\title{
Elevated mature monocytes in bone marrow accompanied with a higher IPSS-R score predicts a poor prognosis in myelodysplastic syndromes
}

An Wu

Ningbo First Hospital

\section{Panpan Gao}

Yuyao People's Hospital of Zhejiang Province

Ningning Wu

Ningbo First Hospital

\section{Cong Shi}

Ningbo First Hospital

\section{Zhenya Huang}

Ningbo First Hospital

Chunmeng Rong

Ningbo First Hospital

Ye Sun

Ningbo First Hospital

\section{Lixia Sheng}

Ningbo First Hospital

Guifang Ouyang ( $\sim$ nbhematology@163.com )

Ningbo First Hospital

\section{Qitian Mu}

Ningbo First Hospital

\section{Research Article}

Keywords: mature monocyte in bone marrow, myelodysplastic syndrome, IPSS-R, prognosis

Posted Date: December 18th, 2020

DOI: https://doi.org/10.21203/rs.3.rs-118785/v1

License: (c) This work is licensed under a Creative Commons Attribution 4.0 International License. Read Full License 


\section{Abstract}

Background: Myelodysplastic syndromes (MDS) is a group of heterogeneous

myeloid clonal diseases originating from hematopoietic stem cells. Clinically, elevated mature monocyte is often observed, but its clinical value still remains unclear.

Methods: We retrospectively analyzed a cohort of 235 MDS patients to explore the prognostic value of the percentage of mature monocyte in bone marrow (PMMBM). All patients were divided into elevated PMMBM group and the normal group by $6 \%$ PMMBM as the cut-off value.

Results: Our results showed that $\mathrm{PMMBM}>6 \%$ was associated with inferior overall survival $(\mathrm{OS})(\mathrm{P}=0.007)$ and leukemia-free survival (LFS) ( $P=0.016)$ along with higher Revised International Prognostic Scoring System (IPSS-R) score $(P<0.0001)$ and higher frequency of IDH2 mutation $(P=0.001)$. Multivariate analyses showed that besides older age (>60 years), lower hemoglobin level $(<10 \mathrm{~g} / \mathrm{dl})$, higher bone marrow blast percentage $(>5 \%)$, poorer karyotype, elevated PMMBM was also an independent adverse prognostic factor for OS in MDS $(P=0.049)$.

Conclusions: These findings indicate that increased PMMBM accompanied with a higher IPSS-R score may predict a poor outcome and provide a novel evaluation factor for MDS patients especially when their karyotype analyses fail.

\section{Background}

Myelodysplastic syndromes (MDS), characterized by ineffective hematopoiesis, manifested by morphologic dysplasia in hematopoietic cells and peripheral cytopenia(s), is a group of heterogeneous myeloid clonal diseases originating from hematopoietic stem cells with a high risk of transforming to secondary acute myeloid leukemia (AML) ${ }^{[1]}$. The prognosis of MDS is extremely heterogeneous due to clinical and biological diversity. Herein, the International Prognostic Scoring System (IPSS) in 1997, the World Health Organization (WHO) Classification-Based Prognostic Scoring System (WPSS) in 2007, the MD Anderson Risk Model Score (MDAS) in 2008 and the Revised IPSS (IPSS-R) in 2012 were introduced to risk-stratify MDS patients ${ }^{[2-5]}$. Recently, lymphocyte-to-monocyte ratio and mutations such as TP53, SRSF2, IDH2 and ASXL1 were also demonstrated to predict the prognosis of MDS ${ }^{[6-10]}$.

Before 2001, chronic myelomonocytic leukemia (CMML) was still categorized into MDS due to its MDS-like characteristics $^{[11]}$. Unlike MDS, CMML is characterized by bone marrow (BM) dysplasia and persistent monocytosis, hence it is placed in a separate category of diseases ${ }^{[12]}$. However, some patients initially manifest MDS characteristics could progress eventually into $\mathrm{CMML}^{[13,14]}$. In general, the prognosis of CMML patients is poorer than MDS, with a median overall survival (OS) of 2 to 3 years and $20 \%$ to $30 \%$ risk of leukemia transformation ${ }^{[15-17]}$. These findings indicate that monocytosis could contribute to progression in MDS.

The monocyte includes monoblast, promonocyte, immature monocyte, and mature monocyte ${ }^{[18]}$, among which monoblast and promonocyte are regarded as "blast" which relates to subtypes in MDS ${ }^{[12]}$. However, clinical value of mature monocyte in BM remains unclear. Hence, 235 MDS patients in our cohort were retrospectively analyzed to measure the prognostic value of the percentage of mature monocyte in bone marrow (PMMBM). Our results suggested that increased PMMBM accompanying with a higher IPSS-R score predicted a poor prognosis in MDS.

\section{Methods}


Clinical and follow-up data of 235 patients were collected who were diagnosed of MDS in Ningbo First Hospital from 2009 to 2018. Diagnosis and classification of MDS and leukemic transformation were made according to the 2016 WHO classification ${ }^{[1]}$. Risk stratifications of MDS were made according to IPSS-R ${ }^{[5]}$. Cases with follow-up for less than 6 months or peripheral blood (PB) monocyte $\geq 1 \times 10^{9} / \mathrm{L}$ were excluded from the analysis. More than half of the patients received symptomatic and supportive treatment. Fifty-three patients $(22.6 \%)$ were treated with intensive chemotherapy, while 17 patients $(7.2 \%)$ received hemopoietic stem cell transplantation (HSCT) and 4 patients (1.7\%), hypomethylating agents. PMMBM accounts for $1 \%-6.2 \%$ of all nucleate cells in normal $\mathrm{BM}^{[19]}$, thus all 235 MDS patients were grouped into two groups basing on 6\% PMMBM as the cut-off value for further analyses. Approval for the retrospective review of these records was obtained from the Ethics Committee of Ningbo First Hospital and was in accordance with the Declaration of Helsinki. Informed consent was obtained from all adult subjects or parents if subjects are under 18.

\section{Morphology analysis}

The morphology of MDS myeloid cells were observed through Wright-Giemsa tinct bone marrow smear. It was evaluated subjectively by light microscopy at low power (10xobjectives) for overall quality and distribution, and then was analyzed at high power (100xoil objectives) for differential count including PMMBM count, with all cells in each containing field counted to maintain representative ratios of cell types ${ }^{[20]}$. All BM morphology findings were interpreted by two experienced and qualified clinical pathologists.

\section{Cytogenetic analysis}

BM cells were collected and cultured in RPMI-1640 medium supplemented with $20 \%$ newborn calf serum for $24 \mathrm{~h}$. Rbanded metaphases were karyotyped according to the International System for Human Cytogenetic Nomenclature (2016) (ISCN2016) ${ }^{[21]}$.

\section{Mutational analysis}

Molecular analysis was performed as a part of the routine clinical work-up. Mutational analysis for 14 common genes of MDS including NRAS, DNMT3A, SF3B1, IDH1, IDH2, TET2, EZH2, JAK2, CBL, ETV6, TP53, SRSF2, ASXL1 and RUNX1 were performed with next generation sequencing.

\section{Statistical Analysis}

Statistical analyses were performed by SPSS 21.0. OS was calculated from the date of initial diagnosis of MDS to the date of death, last follow-up or acquiring allo HSCT. Leukemia-free survival (LFS) was determined from the date of diagnosis to the date of leukemia transformation, last follow-up or acquiring allo HSCT. OS and LFS were analyzed using the Kaplan-Meier method and compared using the log-rank test. Multivariable analyses were used by Cox proportional hazard regression model. Differences in the distribution of continuous variables between categories were analyzed by Mann-Whitney $U$ and categorical variables by Chi-squared test. The receiver operating characteristic (ROC) curve was used to evaluate the diagnostic value of PB monocyte for MDS and the optimal cutoff value was $0.1 \times 10^{9} / \mathrm{L}$ (data not shown). A P value of $<0.05$ was considered statistically significant.

\section{Results}

Patients characteristics 
A total of 235 patients of MDS including 106 females and 129 males were identified over a 10-year period with a median age of 61 years (range 16-90 years). Among these MDS patients, the median OS was 37 months (range 1-125 months, 95\% Cl 25.6-48.4 months) and 30 patients (12.8\%) progressed to AML. Basing on the 2016 WHO classification, all patients were classified as MDS as follows: 31 (13.2\%) MDS-SLD, 64 (27.2\%) MDS-MLD, 15 (6.4\%) MDS-RS, 106 (45.1\%) MDS-EB, 1 (0.4\%) MDS-del(5q), 18 (7.7\%) MDS-U. Besides, 201 patients were stratified into IPSS-R risk groups as follows: 14 (7.0\%) very low, 46 (22.9\%) low, 66 (32.8\%) intermediate, 37 (18.4\%) high and 38 (18.9\%) as very high. Of these, the median IPSS-R score was 4.0(1.0-10.0) and the average score was 4.5. Further information was provided in Table 1.

ble 1. Characteristics of 235 patients with primary MDS 


\begin{tabular}{|c|c|c|c|c|}
\hline able & All patients & $\begin{array}{l}\text { Elevated PMMBM } \\
\text { group }\end{array}$ & Normal group & P-value \\
\hline years median (range) & $61(16-90)$ & $62(24-83)$ & $61(16-90)$ & 0.854 \\
\hline ə/Female, n & $129 / 106$ & $29 / 14$ & $100 / 92$ & 0.067 \\
\hline blast, \% median (range) & $4.0(0-19.5)$ & $9.0(0-19.5)$ & $3.0(0-19.5)$ & $<0.0001$ \\
\hline \multicolumn{5}{|l|}{ 1BM, \% median (range) } \\
\hline & $3.5(0-24.0)$ & $8.0(6.5-24.0)$ & $3.0(0-6.0)$ & $<0.0001$ \\
\hline \multicolumn{5}{|l|}{ pheral Blood } \\
\hline x $10^{9} / \mathrm{L}$ median (range) & $1.2(0-7.4)$ & $1.1(0-6.6)$ & $1.3(0.1-7.4)$ & 0.102 \\
\hline g/dl median (range) & $7.6(2.2-14.2)$ & $7.9(2.9-13.4)$ & $7.6(2.2-14.2)$ & 0.393 \\
\hline x109 / L median (range) & $52.0(2.0-434.0)$ & $40.0(6.0-434.0)$ & $58.0(2.0-340.0)$ & 0.067 \\
\hline $\begin{array}{l}\text { ocytes, x109/L median } \\
\text { ge) }\end{array}$ & $0.2(0-0.9)$ & $0.3(0-0.9)$ & $0.2(0-0.8)$ & 0.005 \\
\hline$\mu \mathrm{g} / \mathrm{L}$ median (range) & $\begin{array}{l}306.1(5.5- \\
2612.0)\end{array}$ & $303.9(9.5-1447.6)$ & $\begin{array}{l}307.0(5.5- \\
2612.0)\end{array}$ & 0.994 \\
\hline mmol/L median (range) & $8.3(1.8-428.0)$ & $8.3(1.8-25.0)$ & $8.3(2.6-428.0)$ & 0.331 \\
\hline 312, mg/L median (range) & $\begin{array}{l}407.0(1.6- \\
1521.0)\end{array}$ & $405.5(94.0-1500.0)$ & $\begin{array}{l}408.0(1.6- \\
1521.0)\end{array}$ & 0.752 \\
\hline 1G, mg $\square \mathrm{L}$ median (range) & $1.9(0-12.6)$ & $2.1(0.4-5.5)$ & $1.8(0-12.6)$ & 0.285 \\
\hline IU/L median (range) & $\begin{array}{l}207.0(54.0- \\
1083.0)\end{array}$ & $219.0(123.0-789.0)$ & $\begin{array}{l}203.0(54.0- \\
1083.0)\end{array}$ & 0.352 \\
\hline $\begin{array}{l}\text { genetic abnormalities, \% } \\
\text { ) }\end{array}$ & $46.8(94 / 201)$ & $56.8(21 / 37)$ & $44.5(73 / 164)$ & 0.178 \\
\hline , \% (n/n) & $16.0(15 / 94)$ & $9.5(2 / 21)$ & $17.8(13 / 73)$ & 0.565 \\
\hline$\%(\mathrm{n} / \mathrm{n})$ & $28.7(27 / 94)$ & $28.6(6 / 21)$ & $28.8(21 / 73)$ & 0.986 \\
\hline olassification & & & & 0.004 \\
\hline S-SLD, \% (n/n) & $13.2(31 / 235)$ & $9.3(4 / 43)$ & $14.1(27 / 192)$ & \\
\hline S-MLD, \% (n/n) & $27.2(64 / 235)$ & $11.6(5 / 43)$ & $30.7(59 / 192)$ & \\
\hline S-RS, \% (n/n) & $6.4(15 / 235)$ & $0(0 / 43)$ & $7.8(15 / 192)$ & \\
\hline S-EB, \% (n/n) & $45.1(106 / 235)$ & $72.1(31 / 43)$ & $39.1(75 / 192)$ & \\
\hline S-del(5q), \% (n/n) & $0.4(1 / 235)$ & $0(0 / 43)$ & $0.5(1 / 192)$ & \\
\hline $\mathrm{S}-\mathrm{U}, \%(\mathrm{n} / \mathrm{n})$ & $7.7(18 / 235)$ & $7.0(3 / 43)$ & $7.8(15 / 192)$ & \\
\hline
\end{tabular}

\section{;-R cytogenetic}

0.429

y good, \% (n/n)

$0.5(1 / 201) \quad 0(0 / 37)$

$0.6(1 / 164)$ 


\begin{tabular}{llll} 
sd, \% (n/n) & $64.2(129 / 201)$ & $51.4(19 / 37)$ & $67.1(110 / 164)$ \\
ərmediate, \%(n/n) & $20.9(42 / 201)$ & $29.7(11 / 37)$ & $18.9(31 / 164)$ \\
rr, \% (n/n) & $3.5(7 / 201)$ & $5.4(2 / 37)$ & $3.0(5 / 164)$ \\
y poor, \% (n/n) & $10.9(22 / 201)$ & $13.5(5 / 37)$ & $10.4(17 / 164)$ \\
i-R risk & & & 0.004 \\
y low, \% (n/n) & $7.0(14 / 201)$ & $0(0 / 37)$ & $8.5 \%(14 / 164)$ \\
v, \% (n/n) & $22.9(46 / 201)$ & $8.1(3 / 37)$ & $26.2 \%(43 / 164)$ \\
ərmediate, \% (n/n) & $32.8(66 / 201)$ & $29.7(11 / 37)$ & $33.5 \%(55 / 164)$ \\
h, \% (n/n) & $18.4(37 / 201)$ & $32.5(12 / 37)$ & $15.3 \%(25 / 164)$ \\
y high, \% (n/n) & $18.9(38 / 201)$ & $29.7(11 / 37)$ & $16.5 \%(27 / 164)$ \\
;-R score & $4.0(1.0-10.0)$ & $5.5(2.0-10.0)$ & $4.0(1.0-10.0)$ \\
e mutation, \% (n/n) & $70.8(63 / 89)$ & $84.6(11 / 13)$ & $68.4(52 / 76)$ \\
remia transformation, \% & $12.8(30 / 235)$ & $23.3(10 / 43)$ & $10.4(20 / 192)$ \\
) & & & \\
\hline
\end{tabular}

Abbreviations: BM, bone marrow; PMMBM, the percentage of mature monocyte in bone marrow; NE, neutrophil; HB, hemoglobin; PLT, platelet; SF, serum ferritin; FA, folic acid; Vit B12, vitamin B12; $\beta 2-M G, \beta 2$-microglobulin; LDH, lactate dehydrogenase; MK, monosomal karyotype: two or more distinct autosomal chromosome monosomies or one single autosomal monosomy with at least one structural abnormalities in two or more metaphases; CK, complex karyotype: three or more cytogenetic abnormalities; MDS-SLD, MDS with single lineage dysplasia; MDS-MLD, MDS with multilineage dysplasia; MDS-RS, MDS with ring sideroblasts; MDS-EB, MDS with excess blasts; MDS-U, unclassifiable; IPSS-R, Revised International Prognostic Scoring System.

\section{Elevated PMMBM in relation to clinical and laboratory factors}

In our cohort, 235 patients were divided into two groups to analyze the correlation between elevated PMMBM and clinical and laboratory characteristics. It showed that the elevated PMMBM group had significantly higher counts of BM blast $(P<0.0001)$ and PB monocyte $(P=0.005)$ as well as higher IPSS-R score $(P<0.0001)$ and risk distribution in terms of IPSS-R ( $P=0.004)$ compared with the normal PMMBM group. Also, the WHO subtype between these two groups had a significant difference $(P=0.004)$. Furthermore, in the elevated PMMBM group, 6 MDS patients were observed to evolve into CMML. There were no significant differences in other factors between two groups (Table 1).

\section{Elevated PMMBM was accompanied with more mutation of IDH2}

Mutations of 14 genes were detected in 89 patients, 63 (70.8\%) of whom harbored mutations. Eight mutations with a minimum 5\% frequency were identified in 14 genes in which ASXL1 and RUNX1 mutations appeared the most (both 13.7\%) , followed by TET2 mutation (12.6\%), TP53 mutation (11.6\%), SF3B1 mutation (10.5\%), SRSF2 and EZH2 mutations (10.0\%) and ETV6 mutation (7.4\%). The elevated PMMBM group harbored higher ratio of gene mutation in comparison with the normal PMMBM group, but the difference was not statistically significant (84.6\% vs. $68.4 \%$, $\mathrm{P}=0.392$ ). Among these mutations, the elevated PMMBM group showed higher mutation frequency of IDH2 compared with the normal PMMBM group $(36.3 \%$ vs. $1.9 \%, P=0.001)$. 
Compared with the normal PMMBM group, the median OS in the elevated PMMBM group was significantly shorter (17 months vs 39 months, $P=0.007$; Fig $1 \mathrm{~A}$ ). Elevated PMMBM group also had an inferior LFS than the normal PMMBM group ( $\mathrm{P}=0.016$; Fig 1B).

In univariate analysis, OS was adversely associated with older age ( $>60$ years) $(P<0.0001)$, higher-risk IPSS-R $(P<0.0001)$, higher BM blast percentage $(>5 \%)(P<0.0001)$, lower hemoglobin $(H B)(<10 \mathrm{~g} / \mathrm{dl})(\mathrm{P}<0.0001)$, neutrophil (NE) $\left(<0.8 \times 10^{9} / \mathrm{L}\right)(\mathrm{P}=0.019)$ and $\mathrm{PB}$ monocyte counts $\left(<0.1 \times 10^{9} / \mathrm{L}\right)(\mathrm{P}=0.021)($ Table 2$)$.

Table 2. Univariate and multivariate analyses for overall survival and leukemia-free survival in 235 patients with primary MDS

\begin{tabular}{|c|c|c|c|c|}
\hline Variables & $\begin{array}{l}\text { Univariate } \\
\text { analysis for OS } \\
\text { P-value }\end{array}$ & $\begin{array}{l}\text { Multivariate } \\
\text { analysis for OS } \\
\text { P-value }\end{array}$ & $\begin{array}{l}\text { Univariate analysis } \\
\text { for LFS } \\
\text { P-value }\end{array}$ & $\begin{array}{l}\text { Multivariate } \\
\text { analysis for LFS } \\
\text { P-value }\end{array}$ \\
\hline Age $\geq 60$ (years) & $<0.0001$ & $<0.0001$ & 0.383 & - \\
\hline Gender (male) & 0.002 & 0.073 & 0.064 & 0.083 \\
\hline $\mathrm{HB}<10 \mathrm{~g} / \mathrm{dl}$ & $<0.0001$ & 0.047 & 0.890 & - \\
\hline $\mathrm{NE}<0.8 \times 10^{9} / \mathrm{L}$ & 0.019 & 0.586 & 0.006 & 0.535 \\
\hline $\mathrm{PLT}<100 \times 10^{9} / \mathrm{L}$ & 0.075 & 0.169 & 0.056 & 0.138 \\
\hline $\begin{array}{l}\text { Monocyte }>0.1 \mathrm{x} \\
10^{9} / \mathrm{L}\end{array}$ & 0.021 & - & - & - \\
\hline BM blast > 5\% & $<0.0001$ & $<0.0001$ & $<0.0001$ & $<0.0001$ \\
\hline $\begin{array}{l}\text { IPSS-R, cytogenetic } \\
\text { risk group }\end{array}$ & $<0.0001$ & 0.002 & 0.051 & 0.898 \\
\hline IPSS-R, risk category & $<0.0001$ & - & $<0.0001$ & - \\
\hline $\begin{array}{l}\mathrm{PMMBM} \leq 6 \% \\
\mathrm{PMMBM}>6 \%\end{array}$ & 0.007 & 0.049 & 0.016 & 0.784 \\
\hline
\end{tabular}

Abbreviations: HB, hemoglobin; NE, neutrophil; PLT, platelet; BM, bone marrow; IPSS-R, Revised International Prognostic Scori PMMBM, the percentage of mature monocyte in bone marrow.

Multivariate analyses showed that older age ( $>60$ years) $(P<0.0001)$, higher BM blast percentage $(>5 \%)(P<0.0001)$, lower HB counts $(<10 \mathrm{~g} / \mathrm{dl})(P=0.047)$ and poorer karyotype $(P=0.002)$ were adverse factors and elevated PMMBM was a significant prognostic factor for worse OS $(P=0.049)$ but not for LFS $(P=0.784)$ (Table 2$)$.

\section{Discussion}

In our 235 MDS patients, elevated PMMBM was associated with a higher BM blast percentage at diagnosis, in accordance with higher IPSS-R scores. Our research suggested that elevated PMMBM was an independent adverse prognostic factor for OS. 
A series of studies ${ }^{[13,14,22]}$ have showed that a subgroup of MDS patients can evolve into CMML and present a poor prognosis. E. Schuler et al ${ }^{[23]}$ conducted similar opinion that MDS patients with BM monocytic proliferation exerted CMML-like characteristics more often. However, the effect of elevated PMMBM on the prognosis of MDS remains unclear. To our knowledge, this study is the first to identify elevated PMMBM as the adverse prognostic impact on MDS. Recently, L Saeed et al ${ }^{[6]}$ found that subnormal absolute monocyte count (AMC) of MDS was associated with an adverse OS in univariate analysis but not in multivariate analysis which is in accordance with our results. Nonetheless, elevated PMMBM was associated with adverse OS and LFS in our study.

The BM microenvironment is composed of BM stromal cells, mesenchymal stem cells, vascular endothelial cells, fibroblasts, mononuclear phagocyte system and cytokines ${ }^{[24]}$. Monocytes are essential cellular components of the host defense system. Due to their high plasticity, monocytes are involved in several cancer-associated processes including immune-tolerance, metastatic spread and neoangiogenesis along with $\mathrm{M} 1$ and $\mathrm{M} 2$-like macrophages induction ${ }^{[25,26]}$. Recent studies have found that M2-like macrophages, called tumor-associated macrophages (TAMs), were involved in promoting tumor progression and metastasis by boosting angiogenesis, stimulating tumor cells' proliferation, migration and invasion ${ }^{[27-29]}$. Although TAMs are initially considered to affect solid tumors, they are later found to predict poor outcomes in blood diseases such as lymphoma, leukemia and multiple myeloma ${ }^{[30]}$. But the roles of TAMs in MDS patients have not been fully elucidated. It is also considered that monocytes can contribute to tumor angiogenesis along with vascular endothelial growth factor (VEGF) to help tumor cells to evade the killing effect of immunocytes, and they can impede differentiation, maturation and proliferation of lymphocytes and promote survival of malignant $\mathrm{T}_{\text {cells }}{ }^{[31,32]}$. Thus, we speculate that elevated PMMBM played an important role in the transformation and progression of MDS.

Further, it was demonstrated in our cohort that MDS patients with elevated PMMBM harbored higher BM plast percentage, PB monocyte count and especially IPSS-R score. It is well known that IPSS-R was widely used in measuring the prognosis of MDS since it was introduced in $2012^{[5]}$. Our results showed that elevated PMMBM at the time of diagnosis significantly correlated with inferior outcomes and was closely accompanied with a higher IPSS-R score which is associated with a shorter OS and even a shorter LFS.

In recent ten years, recurrent somatic mutations in more than 50 genes have been demonstrated in $80-90 \%$ MDS ${ }^{[33]}$, some of which are identified to predict the prognosis of this disease ${ }^{[7-10]}$. In our cohort, mutational analyses of 14 genes relevant to MDS were performed in 89 patients and elevated PMMBM patients harbored a higher mutational rate in IDH2 which predicts an adverse outcome in MDS ${ }^{[8,34]}$. This result indicated that elevated PMMBM MDS patients could have distinct characteristics.

\section{Conclusions}

In summary, we demonstrated that elevated PMMBM accompanied with increased IPSS-R score and higher frequency of IDH2 mutation was associated with a poor prognosis. PMMBM as a prognostic factor could provide a convenient for measuring the prognosis of MDS patients especially when their karyotype analysis fails.

\section{Abbreviations}

Myelodysplastic syndromes (MDS)

The percentage of mature monocyte in bone marrow (PMMBM) 
Overall survival (OS)

Leukemia-free survival (LFS)

Revised International Prognostic Scoring System (IPSS-R)

Acute myeloid leukemia (AML)

International Prognostic Scoring System (IPSS)

World Health Organization (WHO)

World Health Organization Classification-Based Prognostic Scoring System (WPSS)

The MD Anderson Risk Model Score (MDAS)

Chronic myelomonocytic leukemia (CMML)

Bone marrow (BM)

Peripheral blood (PB)

Hemopoietic stem cell transplantation (HSCT)

International System for Human Cytogenetic Nomenclature (2016) (ISCN2016)

Receiver operating characteristic curve (ROC)

Hemoglobin (HB)

Neutrophil (NE)

Absolute monocyte count (AMC)

Tumor-associated macrophages (TAMs)

Vascular endothelial growth factor (VEGF)

Platelet (PLT)

Serum ferritin (SF)

Folic acid (FA)

Vitamin B12 (Vit B12)

$\beta 2$-microglobulin ( $\beta 2-\mathrm{MG})$

lactate dehydrogenase (LDH)

Monosomal karyotype (MK)

Complex karyotype (CK) 
MDS with single lineage dysplasia (MDS-SLD)

MDS with multilineage dysplasia (MDS-MLD)

MDS with ring sideroblasts (MDS-RS)

MDS with excess blasts (MDS-EB)

MDS with unclassifiable (MDS-U)

\section{Declarations}

Ethics approval and consent to participate

Approval for the retrospective review of these records was obtained from the Ethics Committee of Ningbo First Hospital (2020-R196) and was in accordance with the Declaration of Helsinki.

Consent for publication

Not applicable

Availability of data and materials

The data that support the findings of this study are available from Ningbo First Hospital but restrictions apply to the availability of these data, which were used under license for the current study, and so are not publicly available. Data are however available from the authors upon reasonable request and with permission of Ningbo First Hospital. An Wu, the first author, should be contacted if someone wants to request the data from this study.

\section{Competing interests}

The authors declare that they have no competing interests.

\section{Funding}

This research was supported by Zhejiang Provincial Natural Science Foundation of China under Grant No.

(LY20H080001 and LY17H160005), the Medical and Health Science and Technology Projects of Zhejiang Province (2019KY158, 2019KY170, and 2019KY171), the National Science Foundation of Ningbo (2018A610390 and 2016A610152), and the Chinese Medicine Science and Technology Plan Project of Zhejiang Province (2015ZZ018).

\section{Authors' contributions}

A.W. collected and analyzed data and was a major contributor in writing the manuscript. L.S., G.O. and Q.M. designed research and reviewed the manuscript. P.G., N.W., C.S., Z.H., C.R. and Y.S. collected data. All authors read and approved the final manuscript.

\section{Acknowledgements}

Not applicable.

Authors' information

Not applicable. 


\section{References}

[1] Arber DA, Orazi A, Hasserjian R, et al. The 2016 revision to the World Health Organization classification of myeloid neoplasms and acute leukemia. Blood. 2016; doi: 10.1182/blood-2016-03-643544.

[2] Greenberg P, Cox C, LeBeau MM, et al. International Scoring System for Evaluating Prognosis in Myelodysplastic Syndromes. Blood. 1997; doi: 10.1182/blood.V89.6.2079.

[3] Malcovati L, Germing U, Kuendgen A, et al. Time-dependent prognostic scoring system for predicting survival and leukemic evolution in myelodysplastic syndromes. J Clin Oncol. 2007; doi: 10.1200/jco.2006.08.5696.

[4] Kantarjian H, O'Brien S, Ravandi F, et al. Proposal for a new risk model in myelodysplastic syndrome that accounts for events not considered in the original International Prognostic Scoring System. Cancer. 2008; doi: 10.1002/cncr.23697.

[5] Schanz J, Tuchler H, Sole F, et al. New comprehensive cytogenetic scoring system for primary myelodysplastic syndromes (MDS) and oligoblastic acute myeloid leukemia after MDS derived from an international database merge. J Clin Oncol. 2012; doi: 10.1200/JC0.2011.35.6394.

[6] L S, MM P, KH B, et al. Prognostic relevance of lymphocytopenia, monocytopenia and lymphocyte-to-monocyte ratio in primary myelodysplastic syndromes: a single center experience in 889 patients. Blood cancer journal. 2017; doi: 10.1038/bcj.2017.30.

[7] Ren Y, Mei C, Ye L, et al. Analysis of clinical and molecular features of MDS patients with complex karyotype in China. Blood Cells Mol Dis. 2019; doi: 10.1016/j.bcmd.2018.11.006.

[8] Tefferi A, Lasho TL, Patnaik MM, et al. Targeted next-generation sequencing in myelodysplastic syndromes and prognostic interaction between mutations and IPSS-R. American journal of hematology. 2017; doi: 10.1002/ajh.24901.

[9] Arbab Jafari P, Ayatollahi H, Sadeghi R, et al. Prognostic significance of SRSF2 mutations in myelodysplastic syndromes and chronic myelomonocytic leukemia: a meta-analysis. Hematology. 2018; doi:

10.1080/10245332.2018.1471794.

[10] Lin P, Luo Y, Zhu S, et al. Isocitrate dehydrogenase 2 mutations correlate with leukemic transformation and are predicted by 2-hydroxyglutarate in myelodysplastic syndromes. J Cancer Res Clin Oncol. 2018; doi: 10.1007/s00432018-2627-3.

[11] Bennett JM, Catovsky D, Daniel MT, et al. Proposals for the classification of the myelodysplastic syndromes. Br J Haematol. 1982; 51(2): 189-199.

[12] Vardiman JW, Harris NL, Brunning RD. The World Health Organization (WHO) classification of the myeloid neoplasms. Blood. 2002; doi: 10.1182/blood-2002-04-1199.

[13] Selimoglu-Buet D, Badaoui B, Benayoun E, et al. Accumulation of classical monocytes defines a subgroup of MDS that frequently evolves into CMML. Blood. 2017; doi: 10.1182/blood-2017-04-779579.

[14] SA W, N G, J C, et al. Chronic myelomonocytic leukemia evolving from preexisting myelodysplasia shares many features with de novo disease. American journal of clinical pathology. 2006; doi: 10.1309/fu04-p779-u310-r3ee. 
[15] Such E, Germing U, Malcovati L, et al. Development and validation of a prognostic scoring system for patients with chronic myelomonocytic leukemia. Blood. 2013; doi: 10.1182/blood-2012-08-452938.

[16] Germing U, Kundgen A, Gattermann N. Risk assessment in chronic myelomonocytic leukemia (CMML). Leuk Lymphoma. 2004; doi: 10.1080/1042819042000207271.

[17] Kulasekararaj AG, Smith AE, Mian SA, et al. TP53 mutations in myelodysplastic syndrome are strongly correlated with aberrations of chromosome 5, and correlate with adverse prognosis. Br J Haematol. 2013; doi: 10.1111/bjh.12203.

[18] Goasguen JE, Bennett JM, Bain BJ, et al. Morphological evaluation of monocytes and their precursors. Haematologica. 2009; doi: 10.3324/haematol.2008.005421.

[19] Wenrong X, Jianzhong W. Clinical Hematologic Examination, 5th edition. Beijing: People's Sanitary Publishing Press; 2012.

[20] Reagan WJ, Irizarry-Rovira A, Poitout-Belissent F, et al. Best practices for evaluation of bone marrow in nonclinical toxicity studies. Toxicol Pathol. 2011; doi: 10.1177/0192623310396907.

[21] McGowan-Jordan J, Simons A, Schmid M. ISCN 2016: An International System for Human Cytogenomic Nomenclature (2016). Switzerland: S. Karger AG; 2016.

[22] Valent P. Oligo-monocytic CMML and other pre-CMML states: Clinical impact, prognostication and management. Best Pract Res Clin Haematol. 2020; doi: 10.1016/j.beha.2019.101137.

[23] E S, F F, B H, et al. Myelodysplastic syndromes without peripheral monocytosis but with evidence of marrow monocytosis share clinical and molecular characteristics with CMML. Leukemia research. 2018; doi:

10.1016/j.leukres.2017.12.002.

[24] Shafat MS, Gnaneswaran B, Bowles KM, et al. The bone marrow microenvironment - Home of the leukemic blasts. doi: 10.1016/j.blre.2017.03.004.

[25] Canè S, Ugel S, Trovato R, et al. The Endless Saga of Monocyte Diversity. Front Immunol. 2019; doi: 10.3389/fımmu.2019.01786.

[26] Geissmann F, Manz MG, Jung S, et al. Development of monocytes, macrophages, and dendritic cells. Science. 2010; doi: 10.1126/science.1178331.

[27] Pollard JW. Tumour-educated macrophages promote tumour progression and metastasis. Nat Rev Cancer. 2004; doi: $10.1038 /$ nrc1256.

[28] Ruffell B, Affara NI, Coussens LM. Differential macrophage programming in the tumor microenvironment. Trends Immunol. 2012; doi: 10.1016/j.it.2011.12.001.

[29] Yao RR, Li JH, Zhang R, et al. M2-polarized tumor-associated macrophages facilitated migration and epithelialmesenchymal transition of HCC cells via the TLR4/STAT3 signaling pathway. World J Surg Oncol. 2018; doi: 10.1186/s12957-018-1312-y.

[30] Petty AJ, Yang Y. Tumor-Associated Macrophages in Hematologic Malignancies: New Insights and Targeted Therapies. Cells. 2019; doi: 10.3390/cells8121526. 
[31] Wilcox RA, Wada DA, Ziesmer SC, et al. Monocytes promote tumor cell survival in T-cell lymphoproliferative disorders and are impaired in their ability to differentiate into mature dendritic cells. Blood. 2009; doi: 10.1182/blood2009-05-220111.

[32] Mantovani A, Allavena P, Sica A, et al. Cancer-related inflammation. Nature. 2008; doi: 10.1038/nature07205.

[33] Hosono N. Genetic abnormalities and pathophysiology of MDS. Int J Clin Oncol. 2019; doi: 10.1007/s10147-01901462-6.

[34] Nazha A, Bejar R. Molecular Data and the IPSS-R: How Mutational Burden Can Affect Prognostication in MDS. Curr Hematol Malig Rep. 2017; doi: 10.1007/s11899-017-0407-9.

\section{Figures}
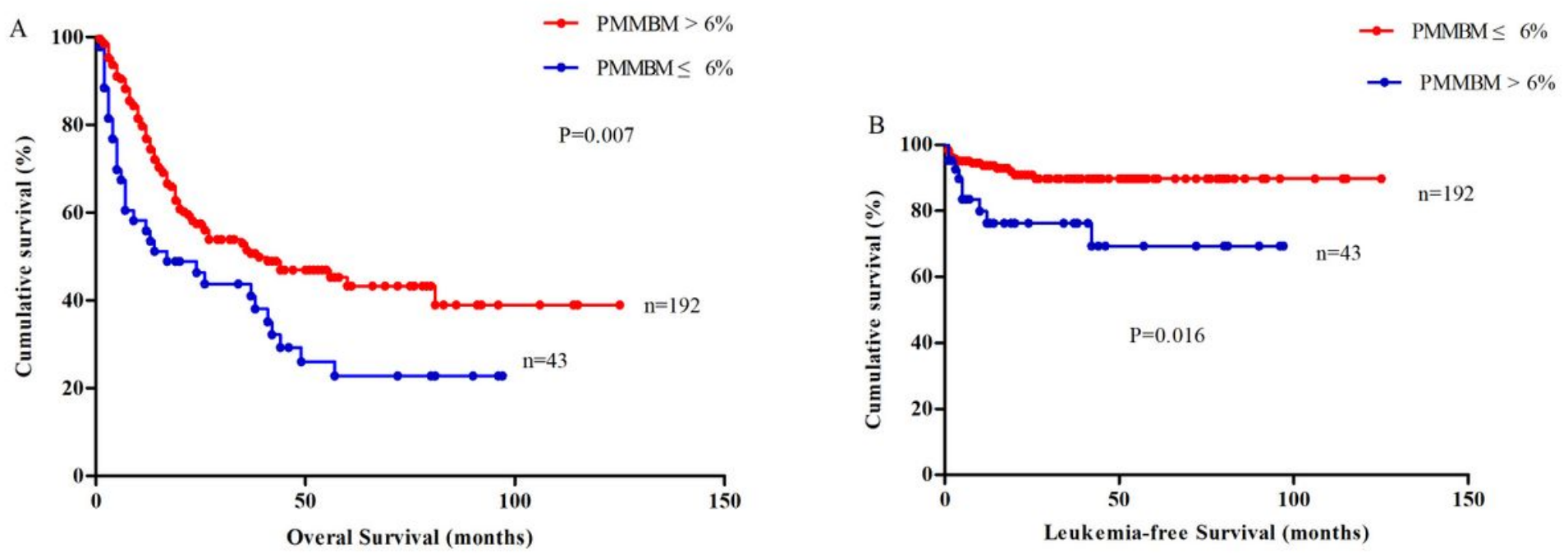

\section{Figure 1}

Overall survival and leukemia-free survival according to PMMBM in MDS (A) Overall survival of 235 patients with primary MDS stratified by $P M M B M \leq 6 \%$ vs $P M M B M>6 \%(P=0.007)$. (B) Leukemia-free survival of 235 patients with primary MDS stratified by $\mathrm{PMMBM} \leq 6 \%$ vs $\mathrm{PMMBM}>6 \%(\mathrm{P}=0.016)$. 